\title{
Chemical Pressure Schemes for the Prediction of Soft Phonon Modes: A Chemist's Guide to the Vibrations of Solid State Materials
}

Joshua Engelkemier and Daniel C. Fredrickson*

Department of Chemistry, University of Wisconsin-Madison, 1101 University Avenue, Madison, WI 53706

*e-mail: danny@chem.wisc.edu

\section{Supporting Information}

\section{S1. Additional Technical Details}

Table S1. Computational parameters and total energies of CP calculations with Abinit

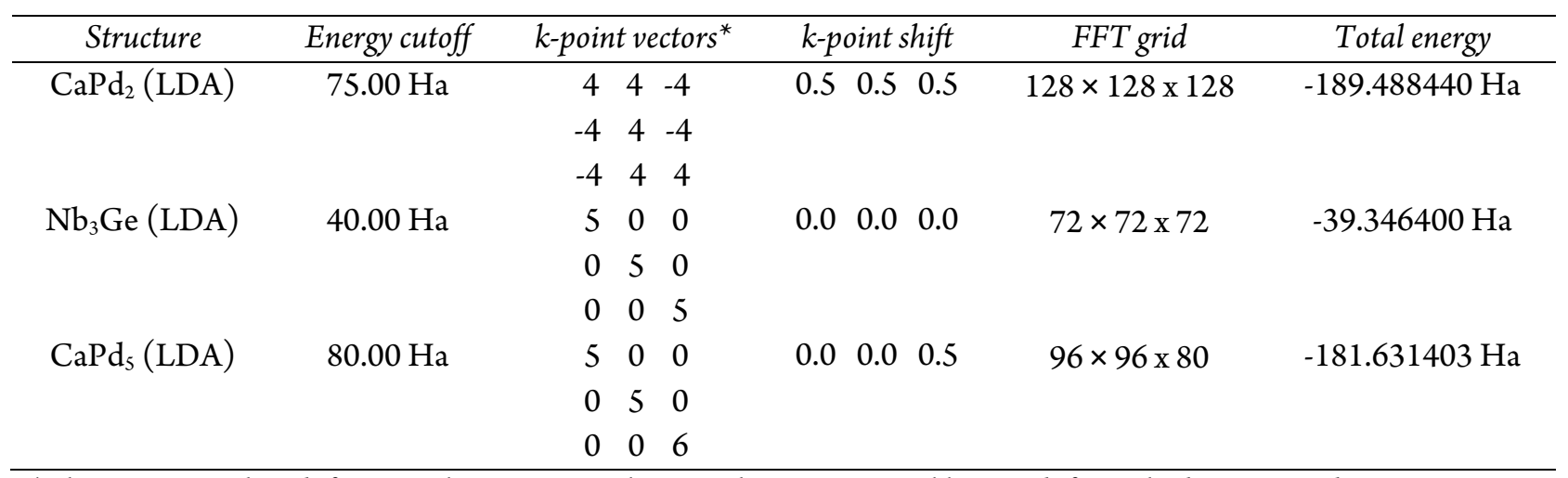

${ }^{*}$ Three vectors that define a real-space super-lattice whose reciprocal lattice defines the k-point grid

Table S2. Computational parameters of response-function calculations with Abinit

\begin{tabular}{cccccc}
\hline Structure & Energy cutoff & k-point grid & k-point shift & FFT grid & q-points \\
\hline $\mathrm{CaPd}_{2}(\mathrm{LDA})$ & $75.00 \mathrm{Ha}$ & $5 \times 5 \times 5$ & 0.00 .00 .0 & $80 \times 80 \times 80$ & 10 \\
$\mathrm{Nb}_{3} \mathrm{Ge}(\mathrm{LDA})$ & $40.00 \mathrm{Ha}$ & $5 \times 5 \times 5$ & 0.00 .00 .0 & $60 \times 60 \times 60$ & 10 \\
$\mathrm{CaPd}_{5}(\mathrm{LDA})$ & $80.00 \mathrm{Ha}$ & $5 \times 5 \times 7$ & 0.00 .00 .0 & $80 \times 80 \times 72$ & 20 \\
\hline
\end{tabular}

Table S3. Computational parameters and total energies of Bader calculations with VASP

\begin{tabular}{cccccc}
\hline Structure & Energy cutoff & k-point grid & FFT grid & Fine FFT grid $^{*}$ & Total energy \\
\hline $\mathrm{CaPd}_{2}$ (PAW-GGA) & $5.17 \mathrm{Ha}$ & $12 \times 12 \times 12$ & $34 \times 34 \times 34$ & $54 \times 54 \times 54$ & $-29.076115 \mathrm{eV}$ \\
$\mathrm{Nb}_{3} \mathrm{Ge}$ (PAW-GGA) & $5.19 \mathrm{Ha}$ & $20 \times 20 \times 20$ & $32 \times 32 \times 32$ & $54 \times 54 \times 54$ & $-72.316164 \mathrm{eV}$ \\
$\mathrm{CaPd}_{5}$ (PAW-GGA) & $5.17 \mathrm{Ha}$ & $19 \times 19 \times 20$ & $36 \times 36 \times 28$ & $50 \times 50 \times 42$ & $-30.297229 \mathrm{eV}$ \\
\hline
\end{tabular}

${ }^{*}$ The second, finer FFT-mesh around the atomic centers in the PAW method 


\section{S2. DFT-Optimized Structural Parameters}

Table S4. Unit cell parameters for DFT-optimized structures (converted to conventional cell for convenience)

\begin{tabular}{ccccccc}
\hline Structure & $a$ & $b$ & $c$ & $a$ & $\beta$ & $\gamma$ \\
\hline $\mathrm{CaPd}_{2}(\mathrm{LDA})$ & $7.56926 \AA$ & $7.56926 \AA$ & $7.56926 \AA$ & $90^{\circ}$ & $90^{\circ}$ & $90^{\circ}$ \\
$\mathrm{CaPd}_{2}($ PAW-GGA) & $7.73955 \AA$ & $7.73955 \AA$ & $7.73955 \AA$ & $90^{\circ}$ & $90^{\circ}$ & $90^{\circ}$ \\
$\mathrm{Nb}_{3} \mathrm{Ge}(\mathrm{LDA})$ & $5.05050 \AA$ & $5.05050 \AA$ & $5.05050 \AA$ & $90^{\circ}$ & $90^{\circ}$ & $90^{\circ}$ \\
$\mathrm{Nb}_{3} \mathrm{Ge}(\mathrm{PAW}-\mathrm{GGA})$ & $5.15611 \AA$ & $5.15611 \AA$ & $5.15611 \AA$ & $90^{\circ}$ & $90^{\circ}$ & $90^{\circ}$ \\
$\mathrm{CaPd} \mathrm{C}_{5}$ (LDA) & $5.25376 \AA$ & $5.25376 \AA$ & $4.38444 \AA$ & $90^{\circ}$ & $90^{\circ}$ & $120^{\circ}$ \\
$\mathrm{CaPd}_{5}($ PAW-GGA) & $5.34490 \AA$ & $5.34490 \AA$ & $4.47471 \AA$ & $90^{\circ}$ & $90^{\circ}$ & $120^{\circ}$ \\
\hline
\end{tabular}

Table S5. Fractional atomic coordinates for Abinit-optimized $\mathrm{CaPd}_{2}$ structure

\begin{tabular}{cccc}
\hline Element & $x$ & $y$ & $z$ \\
\hline $\mathrm{Ca}$ & 0.00000 & 0.00000 & 0.00000 \\
$\mathrm{Ca}$ & 0.50000 & 0.50000 & 0.00000 \\
$\mathrm{Ca}$ & 0.50000 & 0.00000 & 0.50000 \\
$\mathrm{Ca}$ & 0.00000 & 0.50000 & 0.50000 \\
$\mathrm{Ca}$ & 0.25000 & 0.25000 & 0.25000 \\
$\mathrm{Ca}$ & 0.75000 & 0.75000 & 0.25000 \\
$\mathrm{Ca}$ & 0.75000 & 0.25000 & 0.75000 \\
$\mathrm{Ca}$ & 0.25000 & 0.75000 & 0.75000 \\
$\mathrm{Pd}$ & 0.62500 & 0.62500 & 0.62500 \\
$\mathrm{Pd}$ & 0.12500 & 0.12500 & 0.62500 \\
$\mathrm{Pd}$ & 0.12500 & 0.62500 & 0.12500 \\
$\mathrm{Pd}$ & 0.62500 & 0.12500 & 0.12500 \\
$\mathrm{Pd}$ & 0.37500 & 0.37500 & 0.62500 \\
$\mathrm{Pd}$ & 0.87500 & 0.87500 & 0.62500 \\
$\mathrm{Pd}$ & 0.87500 & 0.37500 & 0.12500 \\
$\mathrm{Pd}$ & 0.37500 & 0.87500 & 0.12500 \\
$\mathrm{Pd}$ & 0.37500 & 0.62500 & 0.37500 \\
$\mathrm{Pd}$ & 0.87500 & 0.12500 & 0.37500 \\
$\mathrm{Pd}$ & 0.87500 & 0.62500 & 0.87500 \\
$\mathrm{Pd}$ & 0.37500 & 0.12500 & 0.87500 \\
$\mathrm{Pd}$ & 0.62500 & 0.37500 & 0.37500 \\
$\mathrm{Pd}$ & 0.12500 & 0.87500 & 0.37500 \\
$\mathrm{Pd}$ & 0.12500 & 0.37500 & 0.87500 \\
$\mathrm{Pd}$ & 0.62500 & 0.87500 & 0.87500 \\
\hline & & &
\end{tabular}


Table S6. Fractional atomic coordinates for VASP-optimized $\mathrm{CaPd}_{2}$ structure

\begin{tabular}{cccc}
\hline Element & $x$ & $y$ & $z$ \\
\hline $\mathrm{Ca}$ & 0.00000 & 0.00000 & 0.00000 \\
$\mathrm{Ca}$ & 0.50000 & 0.50000 & 0.00000 \\
$\mathrm{Ca}$ & 0.50000 & 0.00000 & 0.50000 \\
$\mathrm{Ca}$ & 0.00000 & 0.50000 & 0.50000 \\
$\mathrm{Ca}$ & 0.25000 & 0.25000 & 0.25000 \\
$\mathrm{Ca}$ & 0.75000 & 0.75000 & 0.25000 \\
$\mathrm{Ca}$ & 0.75000 & 0.25000 & 0.75000 \\
$\mathrm{Ca}$ & 0.25000 & 0.75000 & 0.75000 \\
$\mathrm{Pd}$ & 0.62500 & 0.62500 & 0.62500 \\
$\mathrm{Pd}$ & 0.12500 & 0.12500 & 0.62500 \\
$\mathrm{Pd}$ & 0.12500 & 0.62500 & 0.12500 \\
$\mathrm{Pd}$ & 0.62500 & 0.12500 & 0.12500 \\
$\mathrm{Pd}$ & 0.37500 & 0.37500 & 0.62500 \\
$\mathrm{Pd}$ & 0.87500 & 0.87500 & 0.62500 \\
$\mathrm{Pd}$ & 0.87500 & 0.37500 & 0.12500 \\
$\mathrm{Pd}$ & 0.37500 & 0.87500 & 0.12500 \\
$\mathrm{Pd}$ & 0.37500 & 0.62500 & 0.37500 \\
$\mathrm{Pd}$ & 0.87500 & 0.12500 & 0.37500 \\
$\mathrm{Pd}$ & 0.87500 & 0.62500 & 0.87500 \\
$\mathrm{Pd}$ & 0.37500 & 0.12500 & 0.87500 \\
$\mathrm{Pd}$ & 0.62500 & 0.37500 & 0.37500 \\
$\mathrm{Pd}$ & 0.12500 & 0.87500 & 0.37500 \\
$\mathrm{Pd}$ & 0.12500 & 0.37500 & 0.87500 \\
$\mathrm{Pd}$ & 0.62500 & 0.87500 & 0.87500 \\
\hline
\end{tabular}

Table S7. Fractional atomic coordinates for Abinit-optimized $\mathrm{Nb}_{3} \mathrm{Ge}$ structure

\begin{tabular}{cccc}
\hline Element & $x$ & $y$ & $z$ \\
\hline $\mathrm{Nb}$ & 0.25000 & 0.00000 & 0.50000 \\
$\mathrm{Nb}$ & 0.75000 & 0.00000 & 0.50000 \\
$\mathrm{Nb}$ & 0.50000 & 0.25000 & 0.00000 \\
$\mathrm{Nb}$ & 0.50000 & 0.75000 & 0.00000 \\
$\mathrm{Nb}$ & 0.00000 & 0.50000 & 0.25000 \\
$\mathrm{Nb}$ & 0.00000 & 0.50000 & 0.75000 \\
$\mathrm{Ge}$ & 0.00000 & 0.00000 & 0.00000 \\
$\mathrm{Ge}$ & 0.50000 & 0.50000 & 0.50000 \\
\hline
\end{tabular}


Table S8. Fractional atomic coordinates for VASP-optimized $\mathrm{Nb}_{3} \mathrm{Ge}$ structure

\begin{tabular}{cccc}
\hline Element & $x$ & $y$ & $z$ \\
\hline $\mathrm{Nb}$ & 0.25000 & 0.00000 & 0.50000 \\
$\mathrm{Nb}$ & 0.75000 & 0.00000 & 0.50000 \\
$\mathrm{Nb}$ & 0.50000 & 0.25000 & 0.00000 \\
$\mathrm{Nb}$ & 0.50000 & 0.75000 & 0.00000 \\
$\mathrm{Nb}$ & 0.00000 & 0.50000 & 0.25000 \\
$\mathrm{Nb}$ & 0.00000 & 0.50000 & 0.75000 \\
$\mathrm{Ge}$ & 0.00000 & 0.00000 & 0.00000 \\
$\mathrm{Ge}$ & 0.50000 & 0.50000 & 0.50000 \\
\hline
\end{tabular}

Table S9. Fractional atomic coordinates for Abinit-optimized $\mathrm{CaPd}_{5}$ structure

\begin{tabular}{cccc}
\hline Element & $x$ & $y$ & $z$ \\
\hline $\mathrm{Ca}$ & 0.00000 & 0.00000 & 0.00000 \\
$\mathrm{Pd}$ & 0.33333 & 0.66667 & 0.00000 \\
$\mathrm{Pd}$ & 0.66667 & 0.33333 & 0.00000 \\
$\mathrm{Pd}$ & 0.50000 & 0.00000 & 0.50000 \\
$\mathrm{Pd}$ & 0.00000 & 0.50000 & 0.50000 \\
$\mathrm{Pd}$ & 0.50000 & 0.50000 & 0.50000 \\
\hline
\end{tabular}

Table S10. Fractional atomic coordinates for VASP-optimized $\mathrm{CaPd}_{5}$ structure

\begin{tabular}{cccc}
\hline Element & $x$ & $y$ & $z$ \\
\hline $\mathrm{Ca}$ & 0.00000 & 0.00000 & 0.00000 \\
$\mathrm{Pd}$ & 0.33333 & 0.66667 & 0.00000 \\
$\mathrm{Pd}$ & 0.66667 & 0.33333 & 0.00000 \\
$\mathrm{Pd}$ & 0.50000 & 0.00000 & 0.50000 \\
$\mathrm{Pd}$ & 0.00000 & 0.50000 & 0.50000 \\
$\mathrm{Pd}$ & 0.50000 & 0.50000 & 0.50000 \\
\hline
\end{tabular}

